v. 8, n. 13, p. 133-144, jan./jun. 2020.

ISSN 2319-0566

DOI: $10.22481 /$ recuesb.v8i13.7101

\title{
ELETROTERAPIA EM PACIENTES COM DOR LOMBAR
}

\section{ELECTROTHERAPY IN INDIVIDUAL WITH BACK PAIN}

\author{
Rafael Tamborela Malheiros ${ }^{1}$ \\ Antonio Carlos Pereira de Araújo ${ }^{2}$ \\ Anna Yasmin Bertão Marques Rodrigues ${ }^{2}$ \\ Eloá Ferreira Yamada ${ }^{3}$ \\ Morgana Duarte da Silva ${ }^{3}$
}

Resumo: A lombalgia crônica acarreta o declínio funcional do paciente e, em geral, seu tratamento é composto por farmacoterapia e fisioterapia. Dentre os recursos fisioterápicos a eletroterapia é uma das técnicas mais utilizadas para a redução da dor. A extensão universitária proporciona ao acadêmico a prática e vivência clínica, ao mesmo tempo, que possibilita a interação com a comunidade. Este trabalho teve por objetivo relatar a experiência do projeto de extensão "Eletroterapia em pacientes com dor lombar". Este estudo é um relato de experiência, em que foram atendidas pessoas com dor lombar crônica no Posto de Saúde 7, da cidade de Uruguaiana/RS. Os participantes eram encaminhados pelo estágio de Fisioterapia em Ortopedia e realizaram 10 sessões, com eletroterapia associada à terapia manual, sendo avaliados pré e pós tratamento quanto à dor e à qualidade de vida. Como resultado, observou-se a redução dos níveis de dor a cada sessão e ao final do tratamento, bem como o aumento da qualidade de vida. Por sua vez, a prática extensionista resultou na integração ensino-serviço através dos atendimentos realizados pelos acadêmicos. Com base no exposto, concluímos que a eletroterapia é um importante recurso na redução da dor, além de apresentar efeitos positivos sobre a qualidade de vida. A extensão universitária foi de extrema importância para os acadêmicos, que vivenciaram um processo que articula o ensino e a pesquisa de forma indissociável, viabilizando a relação transformadora entre Universidade e Sociedade. Palavras-chave: Dor lombar. Dor crônica. Eletroterapia. Extensão.

Abstract: Chronic low back pain leads to the patient's functional decline and, in general, its treatment consists of pharmacotherapy and physiotherapy. Among the physiotherapy resources, electrotherapy is

\footnotetext{
1 Fisioterapeuta e Mestrando em Ciências Fisiológicas, pela Universidade Federal do Pampa (Unipampa), Uruguaiana-RS, Brasil. E-mail: rafaeltmalheiros@gmail.com

${ }^{2}$ Discente do Curso de Fisioterapia, pela Universidade Federal do Pampa (Unipampa), Uruguaiana-RS, Brasil. E-mail: antoniocpa@hotmail.com E-mail: yasminmarques_r@hotmail.com

${ }^{3}$ Docentes do Curso de Fisioterapia, pela Universidade Federal do Pampa (Unipampa), Uruguaiana-RS, Brasil. E-mail:eloayamada@unipampa.edu.br E-mail: morganasilva@unipampa.edu.br
} 
one of the most used techniques for pain reduction. The university extension provides the academic with the practice and clinical experience, at the same time, which enables interaction with the community. This study aimed to report the experience of the electrotherapy extension project in patients with low back pain. People with chronic low back pain were treated at the Health Center 7, in the city of Uruguaiana I RS. Participants were referred by the Physiotherapy internship in Orthopedics and underwent 10 sessions, with electrotherapy associated with manual therapy, being assessed before and after treatment for pain and quality of life. As a result, there was a reduction in pain levels at each session and at the end of treatment, as well as an increase in quality of life. In turn, the extension practice resulted in the teachingservice integration through the assistance provided by the academics. Based on the above, we conclude that electrotherapy is an important resource in reducing pain, in addition to having positive effects on quality of life. University extension was extremely important for academics, who experienced a process that articulates teaching and research in an inseparable way, enabling the transforming relationship between University and Society.

Keywords: Back Pain. Chronic pain. Electrotherapy. Extension.

\section{Introdução}

A dor é um problema de saúde associado às doenças crônicas, gerando altos custos orçamentários para o governo. Um problema comum é a lombalgia crônica, definida pela presença de dor na região lombar com permanência superior a doze semanas e possibilidade de irradiação para membros inferiores (ANDERSSON, 1999; AIRAKSINEN et al., 2006). Esse tipo de sintoma afeta em torno de $80 \%$ da população geral (SILVA; FASSA; VALLE, 2004) e está fortemente relacionada aos fatores psicossociais como medo, angústia/depressão e baixa expectativa de recuperação (HOY et al., 2014). Pessoas com lombalgia apresentam comprometimento funcional, interferindo nas atividades de vida diária (AVD's) e no aumento das taxas de absentismo em todo o mundo (CHOU et al., 2007).

O tratamento conservador, constituído por farmacoterapia e fisioterapia, é o mais aceito para aliviar a dor lombar crônica. A partir do diagnóstico médico, ocorre a prescrição de medicamento, e/ou indicação de atuação de outros profissionais, como o fisioterapeuta (ARAÚJO, 2014) (Figura 1). A terapia farmacológica pode apresentar efeitos adversos como dependência, efeitos indesejados no sistema gastrointestinal, entre outros (JONES et al., 2018) (Figura 1). Com isso, tratamentos não farmacológicos como a fisioterapia podem e devem ser 
usados em indivíduos com dor (CHOU et al., 2018).

O indivíduo com dor lombar pode procurar diretamente o fisioterapeuta, profissional liberal, que faz uso de prestações de serviços com qualificação e competência técnica para atuar em diversos segmentos da saúde dentro de um processo de saúde-doença (REBELATO; BOTOMÉ, 1995; BRASIL, 1969). Existem muitos recursos fisioterápicos utilizados no tratamento da dor lombar como exercícios, cinesioterapia, recursos manuais e recursos térmicos e elétricos - com uso de correntes elétricas de baixa e média frequência (Figura 1). Os recursos usados pelo fisioterapeuta visam ao restabelecimento da saúde do indivíduo, com redução da sintomatologia álgica e retorno das atividades funcionais e laborais do indivíduo (CHOU et al., 2017; AGNE, 2013).

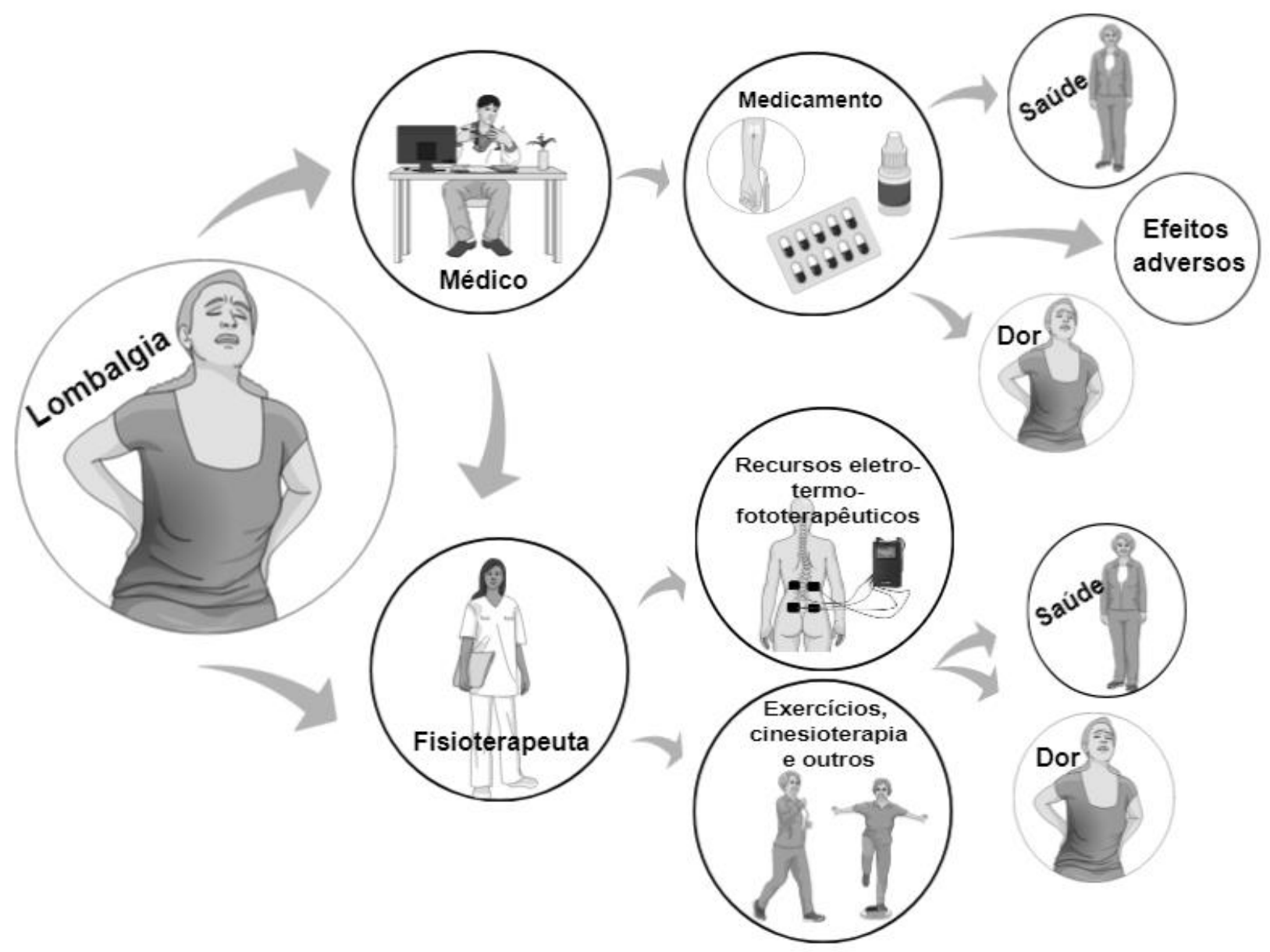

Figura 1: Busca pelo tratamento de lombalgia crônica.

Fonte: Próprio autor. 
Neste contexto, práticas extensionistas realizadas em indivíduos com dor são essenciais na formação do futuro profissional da saúde, proporcionando prática e vivência na assistência, ao mesmo tempo em que se proporciona a comunidade um atendimento especializado que visa à melhora na qualidade vida. Estas ações vão ao encontro do papel transformador da universidade junto à sociedade que está inserida. Desta forma, o acadêmico desenvolve um senso crítico sobre seus estudos - indagando os objetivos e resultados de acordo com a realidade que se encontra. Portanto, os alunos dos cursos de saúde têm a oportunidade de ampliar seu conhecimento técnico e científico com os atendimentos, vivenciando seus direitos e, principalmente, seus deveres como cidadão.

Este trabalho teve por objetivo relatar as ações e atividades desenvolvidas no projeto de extensão "Eletroterapia em pacientes com dor lombar", desenvolvido no curso de Fisioterapia da Universidade Federal do Pampa (UNIPAMPA), campus Uruguaiana (RS/Brasil). O projeto teve como intenção atender pessoas com lombalgia crônica utilizando recursos eletro-termofototerapêuticos associados aos recursos manuais, proporcionando ao acadêmico do curso de Fisioterapia a experiência da extensão universitária, além do contato com os pacientes e realizações dos tratamentos.

\section{Metodologia - Ações e Atividades desenvolvidas no Projeto}

\section{a) Equipe do projeto e pessoas atendidas}

Este estudo é um relato de experiência do projeto de extensão denominado "Eletroterapia em pacientes com dor lombar" foi desenvolvido na Unipampa, em 2014, visando auxiliar na promoção de saúde e qualidade de vida dos pacientes com dores na coluna lombar que procuravam o serviço de Fisioterapia da Universidade, Campus Uruguaiana (RS/Brasil). O grupo de extensão era formado por acadêmicos, fisioterapeutas e professores do curso de Fisioterapia.

As intervenções ocorreram de maio a dezembro de 2014, durante este período foram atendidas pessoas com dor lombar crônica (tempo de dor maior do que três meses) que procuravam o atendimento no estágio de Fisioterapia em Ortopedia que ocorria no Posto de Saúde 7, da cidade de Uruguaiana. A demanda de indivíduos com dor lombar ou lombociatalgia era demasiada e os acadêmicos dos estágios não estavam suprindo essa necessidade. Desta forma,

\footnotetext{
EXTENSÃO \& CIDADANIA

\begin{tabular}{l|ll}
\multirow{2}{*}{ REVISTA } & v. 8, n. 13, p. 133-144, jan./jun. 2020. ISSN 2319-0566
\end{tabular} 
os participantes do projeto faziam uso da eletroterapia com Estimulação Elétrica Nervosa Transcutânea (ou Transcutaneous Electrical Nerve Stimulation, mais conhecida na Fisioterapia como TENS) ou com Corrente Interferencial, associadas à massagem terapêutica da região afetada para alívio da dor dos pacientes, atendendo parte dessa demanda.

Os participantes que tivessem tempo hábil para prosseguir nos atendimentos eram reconduzidos ao estágio para realizar demais terapias, para fortalecimento muscular, melhora da postura ou outro objetivo idealizado após avaliação. Os pacientes portadores de lombalgia participaram de 10 atendimentos, sendo que no primeiro era realizado uma avaliação e posterior tratamento, oito sessões de tratamentos e, no último encontro era realizado tratamento e uma reavaliação, totalizando ao final dez intervenções.

\section{b) Avaliações realizadas}

A avaliação fisioterapêutica foi desenvolvida pelos participantes do projeto e todos os acadêmicos foram treinados para realizar a avaliação de forma padronizada. Esta avaliação foi dividida em quatro etapas descritas a seguir: 1. Anamnese (dados pessoais, histórico de saúde, hábitos de vida e uso de medicamentos); 2. Avaliação física (inspeção, palpação, localização da dor); 3. Avaliação de dor: realizada por meio da Escala Visual Analógica de Dor (EVA); 4. Avaliação da qualidade de vida: utilizou-se o questionário SF-36, que tem como objetivo avaliar a qualidade de vida do indivíduo em relação a alguns aspectos, como capacidade funcional, aspectos físicos, dor, estado geral, vitalidade, aspectos sociais, aspectos emocionais e saúde mental. Os atendimentos foram realizados três vezes por semana e ao final da intervenção realizou-se a reavaliação com o indivíduo.

\section{c) Tratamentos para Lombalgia}

Os acadêmicos participantes do projeto aferiram a pressão arterial e verificavam a EVA de cada participante antes de iniciar o tratamento. Posteriormente, os indivíduos com dor lombar eram orientados a sentar na cadeira de quick massage, os acadêmicos posicionavam a cadeira de forma confortável de acordo com cada voluntário. Posteriormente, os alunos realizavam a higienização do local (com álcool e algodão) onde seriam colocados os eletrodos - quatro eletrodos autoadesivos $(5 \times 5 \mathrm{~cm})$, colocados em igual distância ao lado dos segmentos vertebrais de L1 e S1 (3-4 cm ao lado das vértebras bilateralmente) (Figura 2). Desta forma, foram 
utilizados dois canais do aparelho Neurodyn Ruby Line (Ibramed $\left.{ }^{\circledR}\right)$ com Corrente interferencial (CIF): frequência portadora de $4000 \mathrm{~Hz}, \mathrm{AMF} 10 \mathrm{~Hz}$, Slope 1:1, Sweep $5 \mathrm{~Hz}$, modo varredura automática; ou TENS (Transcutaneous Electrical Nerve Stimulation): frequência de $10 \mathrm{~Hz}$, largura de pulso de $150 \mathrm{~ms}$ - ambas correntes por 30 minutos.

A modalidade de eletroterapia a ser utilizada era definida com base na escolha do profissional/professor em conjunto com o aluno e pela aceitação do paciente ao tipo de corrente, desta forma, o mesmo paciente poderia utilizar de ambas modalidades de eletroterapia durante $o$ período de acompanhamento, embora todos os pacientes tenham optado por permanecer com a mesma durante toda terapia. A intensidade da corrente elétrica foi forte durante toda a terapia, mas sem causar grande desconforto, ajustando-a conforme a sensibilidade de cada indivíduo. Com o término da terapia elétrica, o aparelho foi desligado, os eletrodos retirados e a pele devidamente higienizada.

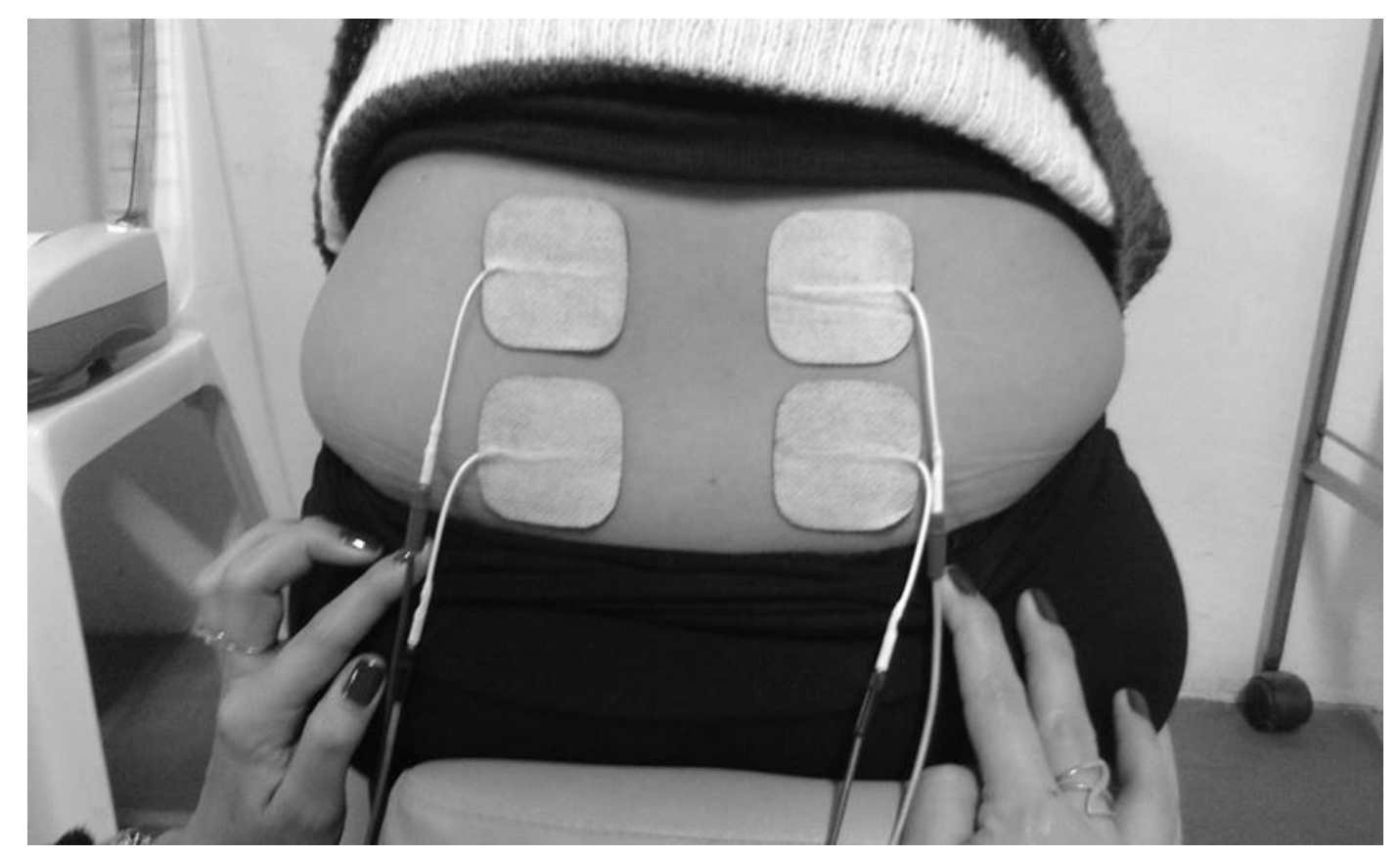

Figura 2: Posicionamento dos eletrodos na região lombar. Fonte: Próprio autor. 
Com o indivíduo na mesma posição, os acadêmicos realizaram manobras manuais sobre a pele na região lombar acometida. Realizando manobras de liberação miofascial seguida de deslizamentos (superficial e profundo), utilizando creme de massagem inodoro, por aproximadamente 10 minutos.

Cessando a terapia, o indivíduo era novamente questionado sobre sua dor (EVA), aferida sua PA e depois liberado. Todos os participantes recebiam orientações posturais e de prática de exercícios adequadamente para evitar a reincidência e/ou agravamento do quadro álgico.

\section{d) Observações gerais}

As terapias foram realizadas no Posto 7 (Uruguaiana), por acadêmicos do curso de Fisioterapia com a supervisão de professores ou fisioterapeutas e tiveram duração de aproximadamente 50 minutos. Os acadêmicos que participaram do projeto receberam treinamento e praticaram entre eles antes de iniciar os tratamentos com os participantes da comunidade. Todos os cuidados com assepsia, uso de luva (quando necessário), vestimentas adequadas, posicionamento das participantes, entre outros, foram utilizados pelos acadêmicos.

Ao final das sessões, os resultados foram demonstrados para os participantes e eles foram encaminhados para o tratamento no Estágio de Fisioterapia em Ortopedia. Ainda, foi realizado uma análise estatística com dados expressos como média \pm erro padrão da média,

analisados utilizando-se teste t não pareado, comparando-se os dados de antes e depois da aplicação da terapia. A análise estatística foi realizada usando o Software GrafhPad (San Diego, CA, USA). O nível de significância em todos os casos foi considerado $\mathrm{p}<0,05$.

\section{Resultados e Discussão}

A partir do projeto de extensão "Eletroterapia em pacientes com dor lombar", foram avaliadas e tratadas dezoito pessoas, porém, quatro delas não realizaram todos os tratamentos por não terem tempo hábil de estar presente na terapia três vezes por semana. Dessa forma, realizaram todas as sessões de tratamento 14 pessoas, sendo 3 homens e 11 mulheres, com idade média 27士51 anos. Em nenhum momento, foram observados sinais vitais grandemente alterados, 
como pressão arterial muito elevada (máxima 155/90mg/hg).

A dor lombar crônica é responsável pela incapacidade funcional do indivíduo, podendo assim ser considerada um fenômeno multidimensional (CROMBIE et al., 1999). A Figura 3 apresenta os resultados quanto à EVA antes e após cada tratamento fisioterapêutico com eletroterapia e massoterapia. Nesta figura, observa-se a redução dos níveis relatados de dor a cada sessão de forma significativa. Além disso, também podemos inferir que da primeira à última sessão houve redução da dor, mesmo antes de iniciar o tratamento no último dia.

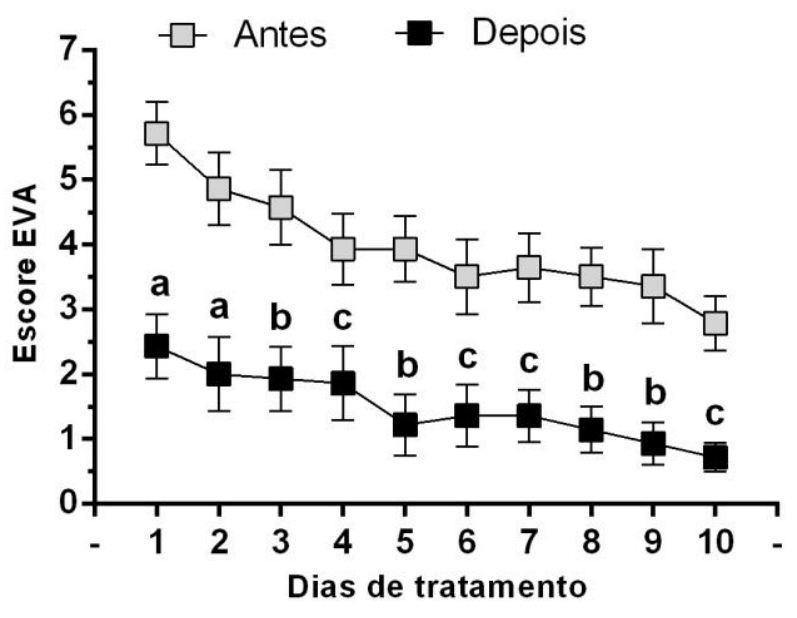

Figura 3: Escala visual da dor relatada pelo indivíduo com dor lombar crônica, antes e depois do tratamento fisioterápico. Cada ponto representa o Escore de EVA de 14 indivíduos. As letras a, b, c demonstram os níveis de significância quando comparados com o valor antes do tratamento, sendo $\mathrm{c}=\mathrm{p}<0,05, \mathrm{~b}=\mathrm{p}<0,001$ e $\mathrm{a}=\mathrm{p}<0,0001$.

Fonte: Próprio autor.

A fisioterapia e a terapia farmacológica são as principais terapêuticas associadas ao tratamento da lombalgia. Devido ao elevado custo monetário do tratamento medicamentoso, bem como seus efeitos adversos, as terapias complementares para o alívio da dor, como o TENS e a CIF, surgem como alternativas de tratamento para esse problema. Acredita-se que os mecanismos de analgesia gerados pela estimulação elétrica estão relacionados ao aumento do limiar de dor devido à interferência causada pela corrente na transmissão das sensações dolorosas. Ainda, alguns estudos apontam que seus efeitos poderiam estar relacionados a um aumento na liberação de opióides endógenos (FACCI et al., 2011; GOMES et al., 2014).

Em revisão na qual foram analisadas diferentes técnicas de tratamento como a 
eletroterapia, terapia manual e cinesioterapia, concluiu-se que a fisioterapia reduziu a dor lombar e apresentou melhora na qualidade de vida dos indivíduos (ARAÚJO; OLIVEIRA; LIBERATORI, 2012). Sabe-se que a dor pode acarretar danos físicos e psicológicos, logo, a analgesia com a eletroterapia pode ter efeito sobre sintomas secundários à dor, como insônia, condições emocionais adversas, atividades de vida diária e, consequentemente, sobre a qualidade de vida do indivíduo. Em nosso estudo, verificamos o aumento do escore de qualidade de vida (Figura 4), o que também foi observado em pesquisa realizada após tratamento fisioterapêutico por TENS, os 14 pacientes com lombalgia apresentaram redução da dor e aumento da qualidade de vida (ALBRECHT; GOULART; WEIS, 2015).
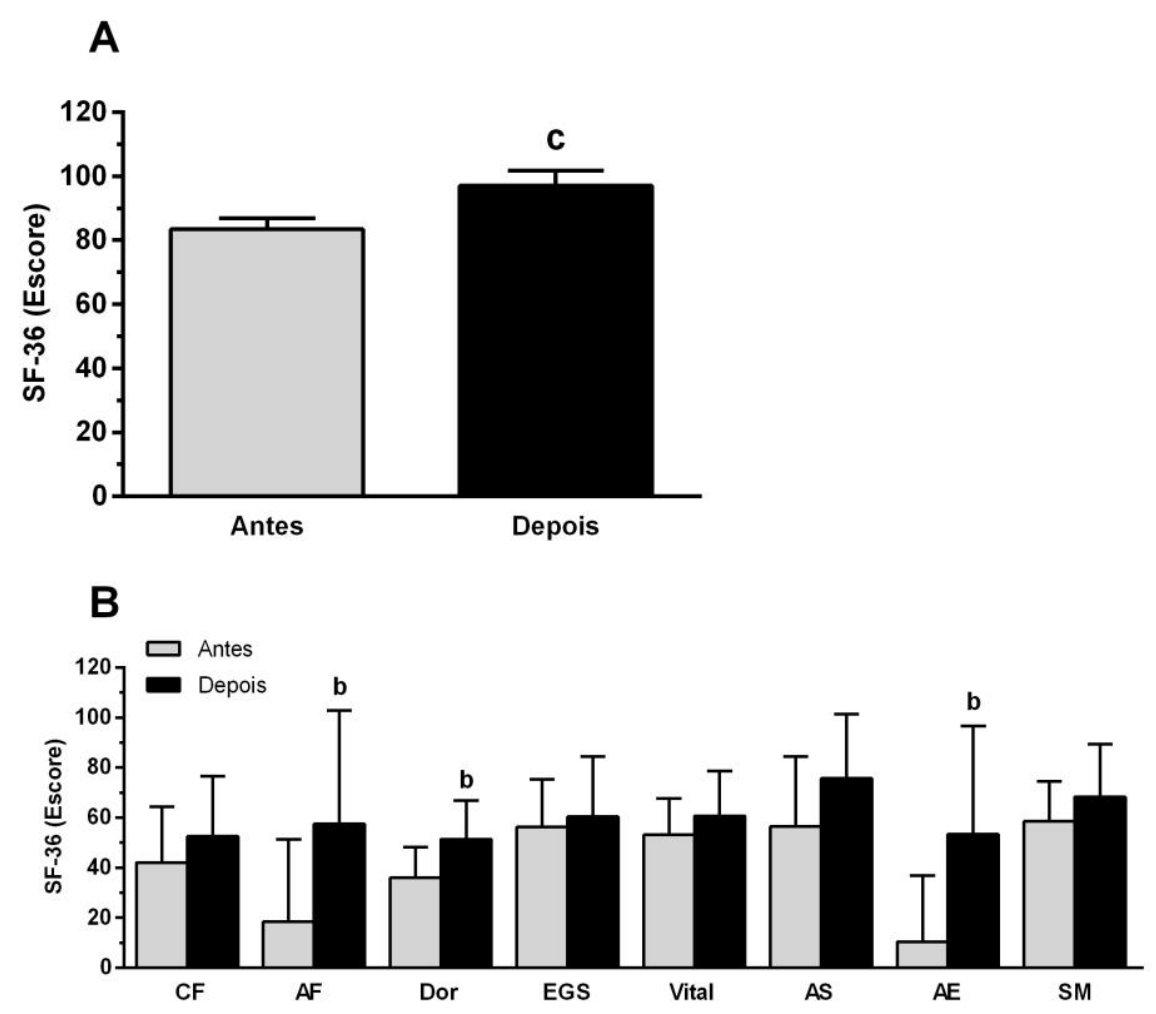

Figura 4: SF-36 realizado no indivíduo com dor lombar crônica, antes e depois do tratamento fisioterápico. A) Escore total do SF-36; B) Escore por domínios do SF-36. Cada coluna representa o Escore do Questionário SF-36 de 14 indivíduos. As letras $(b, c)$ demonstram os níveis de significância quando comparados ao valor antes do tratamento, sendo $c=p<0,05$ e $b=$ $\mathrm{p}<0,001$.

Fonte: Próprio autor.

Sabe-se que a extensão, segundo o Plano Nacional de Extensão, deve ter ações 
transformadoras de impacto social, gerando uma relação dialógica com a sociedade, sociabilizando o ensino e a pesquisa. Logo, os sujeitos devem conviver em situações de interação e cooperação que envolvam o relacionamento entre pessoas ou grupos com experiências diversas, desejos e motivações coletivas. A interação entre universidade e comunidade é fundamental no desenvolvimento de uma sociedade. As atividades desenvolvidas no projeto de extensão "Eletroterapia em pacientes com dor lombar" resultaram na integração ensino-serviço e na atenção em saúde, prestada à comunidade, através dos atendimentos realizados aos pacientes com dor lombar crônica (CARVALHO, 2007).

Além disso, a extensão universitária assume novas percepções e concepções, em que as pessoas da comunidade deixam de ser agentes passivos e passam a ser participantes ativos nesses processos, contribuindo para a construção do conhecimento pelo professor e pelo acadêmico nesta atividade. Os acadêmicos encontraram com acesso ao ensino e prática das atividades do projeto, sem que elas fossem assistencialistas ou mercantilistas, e sim uma comunicação direta com o ensino e a pesquisa desenvolvidas na Unipampa. Ainda, essas práticas didáticas e contextualizadas com a sua própria realidade, favorecem o aprendizado dos temas teóricos/práticos discutidos nos componentes curriculares da graduação - e isso foi muito relatado pelos alunos participantes. Os acadêmicos treinaram sua capacidade de instigar e estimular os pacientes a serem conscientes da sua própria saúde, tornando-se participantes ativos do tratamento e contribuindo para a melhora de sua saúde. Para tanto, os acadêmicos precisaram entender o contexto de vida do paciente, respeitar os direitos sociais deles, educando e protegendo o indivíduo para proporcionar uma melhora da sua saúde.

\section{Conclusão}

Em nosso estudo, observamos que a fisioterapia através das técnicas de eletroterapia por TENS e CIF associados às técnicas manuais, apresentaram redução da dor e do aumento da qualidade de vida dos participantes do projeto de Extensão. A extensão universitária é um processo que articula o ensino e a pesquisa, viabilizando a relação transformadora entre Universidade e Sociedade. Com isso, concluímos que a extensão proporcionou aos acadêmicos 
participantes do projeto a vivência da prática clínica e o entendimento do contexto social dos indivíduos.

\section{Referências}

AGNE, J. E. Eletro termo foto terapia. Santa Maria: O Autor; 2013.

AIRAKSINEN, O. et al. Chapter 4 European guidelines for the management of chronic nonspecific low back pain. European Spine Journal, v. 15, n. 2, p. 192-300, 2006.

ALBRECHT, B. S., GOULART, K. L., WEIS, L. C. Análise da melhora de dor em pacientes com lombalgia submetidos a procedimentos fisioterapêuticos. Revista Jovens Pesquisadores, v. 5, n. 3, p. 17-25, 2015.

ANDERSSON, G.B. Epidemiological features of chronic low-back pain. The Lancet, v. 354, n. 9178, p. 581-585, 1999.

ARAÚJO, A. G. S.; OLIVEIRA, L.; LIBERATORI, M. F.; Protocolo fisioterapêutico no tratamento da lombalgia. Cinergis, v. 13, n. 4, p. 56-63, 2012.

ARAÚJO, A. R. R. M. Trajetória profissional do fisioterapeuta: reconhecimento e interrelações no campo da saúde. Tese (Doutorado em Sociologia) - Universidade Federal da Paraíba, Paraíba, 2014.

BRASIL. Decreto Lei n. 938, de 13 de outubro de 1969. Provê sobre as profissões de fisioterapeuta e terapeuta ocupacional, e dá outras providências. Disponível em: https://www.coffito.gov.br/nsite/?p=3317. Acesso em: 31 maio 2018.

CARVALHO, M. A. P. Construção compartilhada do conhecimento: análise da produção de material educativo. In: BRASIL. Ministério da Saúde. Secretaria de Gestão Estratégica e Participativa. Caderno de Educação Popular em Saúde. Brasília-DF: Ministério da Saúde, 2007. p. 91-105.

CHOU, R. et al. Clinical Efficacy Assessment Subcommittee of the American College of Physicians. Diagnosis and treatment of low back pain: a joint clinical practice guideline from the American College of Physicians and the American Pain Society. Annals of Internal Medicine, v. 147, p. 478-491, 2007.

CHOU, R. et al. Nonpharmacologic therapies for low back pain: a systematic review for an American College of Physicians Clinical Practice Guideline. Annals of Internal Medicine, v. 166, n. 7, p. 493-505, 2017. 
CHOU, R. et al. The Global Spine Care Initiative: applying evidence-based guidelines on the non-invasive management of back and neck pain to low- and middle-income communities. European Spine Journal, v. 27, n. 6, p. 851-860, 2018.

CROMBIE, I. K. et al. Epidemiology of pain: a report of the task force on epidemiology. Seattle: IASP Press, 1999.

FACCI, L. M. et al. Efeitos da estimulação elétrica nervosa transcutânea (tens) e da corrente interferêncial (CI) em pacientes com lombalgia crônica não específica: Ensaio clínico randomizado. São Paulo Medical Journal, v. 129, n. 4, p. 206-216, 2011.

GOMES, A. O. et al. As influências de diferentes frequências da estimulação elétrica nervosa transcutânea no limiar e intensidade de dor em indivíduos jovens. Einstein, v. 12, n. 3, p. 318$322,2014$.

JONES, M. R. et al. Pain States, the Opioid Epidemic, and the Role of Radiologists. Current Pain and Headache Reports, v. 23, n. 22, p 3-20, 2008.

OY, D. et al. The global burden of low back pain: estimates from the Global Burden of Disease 2010 study. Annals of Rheumatic Diseases, v. 73, p. 968-974, 2014.

REBElATO, J. R., BOTOMÉ, S. P. Fisioterapia no Brasil. São Paulo: Manole, 1995.

SILVA, M. C.; FASSA, A. C. G.; VALLE, N. C. J. Dor lombar crônica em uma população adulta do Sul do Brasil: prevalência e fatores associados. Cadernos de Saúde Pública, Rio de Janeiro, v. 20, n. 2, p. 377-385, 2004.

VERAS, R. P., PARAHYBA, M. I. O anacronismo dos modelos assistenciais para os idosos na área da saúde: desafios para o setor privado. Cadernos de Saúde Pública, Rio de Janeiro, v. 23, n. 10, p. 2479-2489, 2007.

Recebido em: 13 de julho de 2018.

Aceito em: 19 de junho de 2020.

EXTENSÃO \& CIDADANIA 